\title{
Cross-Generational Effects of Discrimination Among Immigrant Mothers: Perceived Discrimination Predicts Child's Healthcare Visits for Illness
}

\author{
May Ling Halim \\ New York University
}

\author{
Hirokazu Yoshikawa \\ Harvard University
}

\author{
David M. Amodio \\ New York University
}

\begin{abstract}
Objective: This study tested whether an immigrant mother's perception of ethnic and language-based discrimination affects the health of her child (indexed by the child's frequency of sick visits to the doctor, adjusting for well-visits), as a function of her ethnic-group attachment and length of U.S. residency. Method: A community-based sample of 98 immigrant Dominican and Mexican mothers of normally developing 14-month-old children were interviewed. Mothers reported their perceived ethnic and language-based discrimination, degree of ethnic-group attachment, length of time in the United States, and frequency of their child's doctor visits for both illness and routine (healthy) exams. Results: Among more recent immigrants, greater perceived ethnic and language-based discrimination were associated with more frequent sick-child visits, but only among those reporting low ethnic-group attachment. The associations between both forms of perceived discrimination and sick-child visits were not observed among mothers reporting high ethnic-group attachment. Among more established immigrants, perceived language-based discrimination was associated with more frequent sick-child visits regardless of ethnic-group attachment. Conclusion: These results suggest that a Latina mother's experience with ethnic and language-based discrimination is associated with her child's health, as indicated by doctor visits for illness, but that strong ethnic-group attachment may mitigate this association among recent immigrants.
\end{abstract}

Keywords: immigration, stigma, ethnic attachment, health, prejudice

Immigrants often face several challenges as they attempt to integrate with members of their host country. One of these is discrimination, which may be based on the color of their skin, the way they speak, or their membership in a particular ethnic or national group (Deaux, 2006). Regardless of its basis, discrimination is a major component of the acculturative stressors that immigrants typically experience when they move to a new country

This article was published Online First March 5, 2012.

May Ling Halim, Department of Psychology, New York University; Hirokazu Yoshikawa, Graduate School of Education, Harvard University; David M. Amodio, Department of Psychology and Center for Neural Science, New York University.

The data reported in this article were collected as part of a study conducted at New York University's Center for Research on Culture, Development, and Education (CRCDE), within the Department of Applied Psychology at NYU's Steinhardt School of Culture, Education, and Human Development. This research was supported by the National Science Foundation grants BCS \#021859 and IRADS \#0721383 to Hirokazu Yoshikawa and BCS \#0847350 to David Amodio. The authors thank the staff and volunteers at the CRCDE, the families who participated, Sean Lane and Patrick Shrout for their assistance with statistical procedures, Jill Swencionis and Sophie Wharton for their help in preparing the manuscript, and David Chae for his feedback on earlier versions of this article.

Correspondence concerning this article should be addressed to David M. Amodio, Department of Psychology, New York University, 6 Washington Place, New York, NY 10003. E-mail: david.amodio@nyu.edu (de Snyder, 1987). Importantly, these types of discrimination have been associated with a host of negative mental and physical health outcomes, such as increased psychological distress (e.g., Moradi \& Risco, 2006), worse physical health (Finch, Hummer, Kolody, \& Vega, 2001), and increased rates of risky health behaviors (e.g., Yoshikawa, Wilson, Chae, \& Cheng, 2004).

Although much past research has found a link between discrimination and health (Pascoe \& Richman, 2009), emerging research suggests that a mother's psychological response to discrimination may also have implications for the health of her child. High levels of perceived stress during pregnancy are associated with health risks for infants (Rich-Edwards \& Grizzard, 2005; Wright, 2011). Given the link between discrimination and stress, these findings raise the possibility that the negative health implications of discrimination may extend beyond the mother to her child as early as the first year of life-a pernicious example of how discrimination may have enduring effects on its victims across generations. Indeed, racial and ethnic discrimination has been identified as a risk factor for low-weight or premature births, even after adjusting for socioeconomic status (Giscombe \& Lobel, 2005). Among African American mothers, stress responses associated with perceived discrimination predicted newborn low birth weight (Dominguez, Dunkel-Schetter, Glynn, Hobel, \& Sandman, 2008). Low birth weight, in turn, is associated with many health risks and developmental problems across the life span (McCormick, 
Brooks-Gunn, Workman-Daniels, Turner, \& Peckham, 1992). These findings suggest that greater perceived discrimination in a mother may be associated with poorer health in her child.

The current study examined this hypothesized relationship among Latina immigrants. Latinos are the largest and fastest growing ethnic minority group in the United States (U.S. Census, 2011), and the need for research on the health of Latino children has been named an urgent public health priority (Flores et al., 2002). Yet few studies have examined the effects of perceived discrimination on health among Latino samples (exceptions: Finch et al., 2001; Finch \& Vega, 2003; Gee, Ryan, LaFlamme, \& Holt, 2006; Stuber, Galea, Ahern, Blaney, \& Fuller, 2003). Like African Americans, Latinos often experience discrimination on the basis of their ethnicity. However, because Latino immigrants come from Spanish-speaking countries, they also experience discrimination due to their difficulty with spoken and written English. Although past work has examined the relation between language proficiency and access to medical care (Timmins, 2002), language-based discrimination is an understudied, yet potent, form of discrimination that is particularly relevant to this population. Past research suggests that language-based discrimination is conceptually distinct from ethnic discrimination, as it has been linked independently to negative health outcomes (Spencer \& Chen, 2004; Yoo, Gee, \& Takeuchi, 2009). Therefore, it was important in the present research to investigate the effects of language-based discrimination in addition to ethnic discrimination.

For new immigrants, connections with other members of their ethnic group provide an important source of social support, and thus an immigrant's sense of attachment to her ethnic group may moderate the effect of perceived discrimination on child health in Latina immigrant mothers. Ethnic attachment refers to an individual's sense of belonging and engagement with members of her own ethnic group (Phinney, 1992). ${ }^{1}$ In the present study, ethnic attachment was expected to attenuate the effect of perceived discrimination on a child's health for two reasons. First, stronger ethnic-group attachment has been associated with greater social support and connectedness to other members of one's ethnic group (Kim, 1999), especially among Latina women (Deaux \& Martin, 2003). The salubrious and protective role of social support has been shown in other samples, too, such as in former Soviet Union immigrants to Finland (Jasinskaja-Lahti, Liebkind, Jaakkola, \& Reuter, 2006). Second, individuals with stronger ethnic attachment are more likely to possess traditional Latino cultural values, such as familism (an emphasis on family and kinship), religiosity, and more traditional norms, which are related to better health practices (Hayes-Bautista, 2002). Given these findings, a mother's strong ethnic attachment was hypothesized to buffer the effects of perceived discrimination on her child's health.

The attenuation of the effects of perceived discrimination on children's health due to a mother's ethnic attachment may be more or less pronounced depending on the mother's length of U.S. residency. Indeed, greater social support has been associated with higher infant birth weight for foreign-born, but not for U.S.-born, Latina women (Campos et al., 2008). Ethnic attachment has also been shown to decrease general acculturative stress, particularly among recent immigrants (Deaux, 2006). Because language issues and ethnic-group attachment are especially important among newer immigrants, the effects of these factors on a child's health were expected to be more pronounced among mothers who had more recently moved to the United States. Thus, strong ethnicgroup attachment was hypothesized to mitigate the effects of perceived discrimination on health primarily among newer immigrants.

The current study examined the effects of mothers' perceived ethnic and language-based discrimination on the health of their children, as a function of ethnic-group attachment and length of residency in the United States. The sample included immigrant mothers from Mexico and the Dominican Republic who had 14month-old children. These mothers were interviewed about their perceptions of discrimination, their ethnic-group attachment, and the number of doctor visits made in the previous year for both regular check-ups and for their child's illness. The frequency of the sick-child visits, after adjusting for well-child visits (i.e., regular check-ups), was used as a proxy for child's health. Sick-child visits have been used as an objective indicator of children's health in intervention-based research (Phillips-Howard et al., 2003) and have been correlated with children's actual illnesses (e.g., Wright et al., 1981). Greater discrimination was hypothesized to predict more sick-child visits, after adjusting for well-child visits, but high ethnic attachment was hypothesized to attenuate this relation, especially among recent immigrants. Both ethnic and languagebased discrimination were expected to be associated with sickchild visits, but given the specific challenges of Latina immigrants, language-based discrimination was expected to be especially relevant.

\section{Method}

\section{Participants and Procedure}

Participants included 98 mothers $(M$ age $=27.19$ years, $S D=$ 5.56) of normally developing 14-month-olds who were born as healthy, full-term infants $(M=14.73$ months, $S D=1.25$; 51 girls, 44 boys). All participants were first generation immigrants (i.e., foreign-born) from Mexico $(n=53)$ or the Dominican Republic $(n=42)$, two of the largest Latino immigrant groups in the United States, and the two largest in New York City. Participants were recruited at the maternity wards of New York City hospitals to take part in a larger study on culture and learning. Participants were eligible if they (1) were $18+$ years of age, (2) were not living in a shelter, and (3) self-identified as Mexican or Dominican. In the recruiting phase of this project, researchers approached a total of 560 Dominican and Mexican women. Of these, 32\% agreed to participate, $43 \%$ were not interested, $15 \%$ were ineligible, and $10 \%$ were unable to be reached after initial contact. By the time children were 14 months, the attrition rate of mothers was $47 \%$. Attrition analyses examining a number of characteristics found no significant differences between mothers with and without data at baseline versus at 14 months.

Data were excluded from 3 mothers of children with an extreme number of sick-child visits (10 or more; greater than 3 SDs from

\footnotetext{
${ }^{1}$ Ethnic attachment is distinct from the more widely-studied construct of ethnic identity centrality (i.e., the importance one's ethnic identity; see Pascoe \& Richman, 2009), for which there is more debate concerning the moderating effect on the link between discrimination and health (Yip, Gee, \& Takeuchi, 2008).
} 
mean), leaving 95 families for analysis. Of these excluded participants, two mothers reported 15 visits and one reported 14 visits. Although the inclusion of these outliers did not change the pattern of results, these were special cases of chronic illness and deemed inappropriate for inclusion.

A female researcher (European American or Latina) interviewed participants in their preferred language. Although all participants were native Spanish speakers, $84.2 \%$ preferred to be interviewed in Spanish, whereas $15.8 \%$ preferred to be interviewed in English. Bilingual staff translated all instruments into Spanish, and then back-translated them into English. Multiple meetings were held with a team of researchers to make sure that all questions were understandable and that the meaning of each question was intact across languages. Scores on measures did not differ as a function of language, with the unsurprising exception of language-based discrimination; interviews conducted in Spanish ( $n=80, M=.59$, $S D=.59$ ) yielded greater reported language-based discrimination than those conducted in English $(n=13, M=.22, S D=.50)$, $t(91)=2.13, p<.05, d=.45$.

\section{Measures}

Child's doctor visits. To assess the extent to which mothers took their child to the doctor for concerns about illness (sick visits), interviewers asked mothers, "How many times in the past year did you take [your baby] to a doctor because he or she was sick?" To adjust for differences in health care utilization, the frequency of well-child visits to the doctor was also assessed by asking mothers, "How many times since your child was born did you take him/her when he or she was well (not sick) to a doctor for a regular check-up (well-baby visit)?"

Perceived ethnic discrimination. Mothers' perceptions of ethnic discrimination were assessed using six items adapted from the Everyday Discrimination Scale (Williams, Yu, Jackson, \& Anderson, 1997), including, "How often have you (been treated with less courtesy than other people/had trouble finding a job/ received poorer service than other people at stores, restaurants, or banks/been treated poorly at work by coworkers/been treated poorly at work by supervisors/been treated poorly by the police) because you are (ethnicity)?" (0, Never, to 3, Often; $\alpha=.75)$. Item wording was adapted slightly to be more sensitive to ethnic discrimination experiences and stereotypes of Latina immigrant women in the sample.

Perceived language-based discrimination. Mothers' perceptions of language discrimination were assessed using six items adapted from the perceived everyday ethnic discrimination items, with the substitution of ". . . because of how you wrote or spoke English" for "... because you are (ethnicity)" (e.g., "How often have you been treated with less courtesy than other people because of how you wrote or spoke English?") $(\alpha=$.78). Perceived language-based discrimination was positively correlated with perceived ethnic discrimination, $r(93)=.71, p<.001$. Data from two mothers were missing on this measure and thus not included in analyses involving this variable.

Mother's ethnic-group attachment. Mothers' ethnic-group attachment was measured using a scale of two items: "I have a strong sense of belonging to my ethnic group," and "I feel a strong attachment to my ethnic group", $r(94)=.68, p<.001$. These two items were taken from the affirmation and belonging subscale of the Ethnic Identity Scale (Phinney, 1992).

Years of U.S. residency. The length of time since mothers' initial immigration to the United States was assessed by asking, "How many years have you been in the U.S.?"

Demographic variables. Indicators of socioeconomic status were collected at the time of the child's birth, by interviewers as described above, to be included as covariates. Mothers reported annual household income and education level. See Table 1. Mother's education level was treated as a categorical variable (completed some college vs. only completed high school or received a GED vs. did not complete high school) and dummy coded into two variables, with those reporting less than a high school education as the reference group. Additional covariates included marital status, "well-child" visits (i.e., healthy visits) to the doctor, and child age in months. Ethnic group was included as a dummy variable in all analyses with Mexican families as the reference group, although this difference was not expected to account for the hypothesized effects.

Mother's response to her child's illness. To clarify the meaning of sick-child doctor visits, interviewers asked mothers,

Table 1

Descriptive Statistics for Key Variables

\begin{tabular}{|c|c|c|c|c|}
\hline Measure & Full sample $M(S D)$ & Dominican $M(S D)$ & Mexican $M(S D)$ & Range (full sample) \\
\hline Sick-child visits & $3.14(2.36)$ & $3.38(2.56)$ & $2.94(2.20)$ & $.00-10.00$ \\
\hline \multicolumn{5}{|l|}{ Mother variables } \\
\hline Perceived ethnic discrimination* & $.48(.52)$ & $.31(.35)$ & $.61(.58)$ & $.00-2.00$ \\
\hline Perceived language-based discrimination & $.54(.59)$ & $.46(.56)$ & $.60(.61)$ & $.00-2.17$ \\
\hline Ethnic-group attachment & $3.45(.59)$ & $3.46(.46)$ & $3.43(.67)$ & $1.00-4.00$ \\
\hline Years of U.S. residency & $8.42(5.71)$ & $9.12(6.03)$ & $7.87(5.44)$ & $.00-24.00$ \\
\hline \multicolumn{5}{|l|}{ Covariates } \\
\hline Well-child visits & $6.36(2.20)$ & $6.60(2.25)$ & $6.17(2.17)$ & $2.00-14.00$ \\
\hline Average annual household income & $\$ 22,190(\$ 15,382)$ & $\$ 21,884(\$ 12,216)$ & $\$ 22,432(\$ 17,604)$ & $\$ 0-\$ 96,000$ \\
\hline Married & $45 \%$ & $43 \%$ & $47 \%$ & N/A \\
\hline Mother high school education or beyond* & $57 \%$ & $74 \%$ & $47 \%$ & N/A \\
\hline \multicolumn{5}{|l|}{ Other demographic variables } \\
\hline Preferred interview to be conducted in Spanish* & $84 \%$ & $71 \%$ & $94 \%$ & N/A \\
\hline
\end{tabular}

Note. Asterisks $\left({ }^{*}\right)$ indicate a significant difference between Dominican and Mexican participants at the $p<.05$ level. 
"What did you do the last time your child was sick?" Their response options included: (1) brought child to the hospital or ER or to a clinic at a hospital, (2) brought child to a clinic not in a hospital, (3) brought child to a private doctor's office not in a clinic, (4) gave home remedies, and (5) kept child home. Responses 1-3 were categorized as going to a doctor and 4-5 together as giving home remedies or staying at home.

\section{Statistical Analysis Approach}

Generalized estimating equations (GEE; Zeger, Liang, \& Albert, 1988) were used to test the hypotheses predicting sick-child visits because this dependent measure represents a count variable (i.e., number of sick visits). GEEs permit a regression model to be analyzed with a Poisson distribution, which is characteristic of many count variables, using a log link function. Predictor variables included mother's perceived discrimination (ethnic and languagebased discrimination in two separate equations), mother's ethnicgroup attachment, mother's length of stay in the United States, and all higher-level interactions among these three variables, entered simultaneously in the regression model. Adjustment variables (income, education, marital status, number of well-child visits, child age, and ethnicity) were included as covariates. All continuous predictor variables were mean-centered.

To test whether there were differences between Mexican and Dominican families in the relations among key variables of interest, interaction predictor variables involving ethnicity were included in the GEEs (ethnicity $\times$ discrimination, ethnicity $\times$ ethnic-group attachment, etc., and all three- and four-way interactions). These analyses revealed no significant interactions with ethnicity; thus, data from the two groups were combined for the final set of analyses.

\section{Results}

Means and standard deviations for all variables of interest are reported in Table 1, and intercorrelations among all measures are reported in Table 2. An examination of demographic variables indicated that Mexican and Dominican families did not differ significantly in annual household income, proportion of married parents, generational status, or number of well-child visits. How- ever, Mexican mothers were more likely than Dominican mothers to report that Spanish was their dominant language, $\chi^{2}(1, N=$ $95)=9.25, p<.01, \varphi=.31$. Also, average education level was higher among Dominican mothers than Mexican mothers, $\chi^{2}(1$, $N=95)=16.56, p<.001, \varphi=.42$. See Table 1 .

Dominican and Mexican mothers did not differ in their reported frequency of sick-child doctor visits, perceived language-based discrimination, level of ethnic-group attachment, or length of U.S. residency (see Table 1). However, Mexican mothers reported a higher degree of ethnic discrimination than did Dominican mothers, $t(93)=3.00, p<.01, d=.62$.

\section{Mothers' Perceived Ethnic Discrimination and Children's Health}

It was hypothesized that greater perceived ethnic discrimination among mothers would predict more children's sick visits to the doctor, but that ethnic-group attachment would attenuate this relation, especially among recent immigrants. The GEE analysis revealed a significant main effect for ethnic-group attachment, as well as a significant ethnic-group attachment by years in U.S. interaction. However, these effects were qualified by the predicted significant three-way interaction (see Table 3).

To interpret the three-way interaction, the effects of perceived ethnic discrimination and ethnic-group attachment were examined separately for recent versus established immigrants (at $+/-1 S D$ relative to the mean values). For recent immigrants, this two-way interaction was significant, $B=-1.02$, $\beta=-.31, t(82)=7.66, p<.01$. Supporting the central prediction, simple slope analyses indicated that greater perceived discrimination predicted more frequent sick-child visits among those with low ethnic-group attachment, $B=.61, \beta=$ $.32, t(82)=10.00, p<.01$. This relationship was marginally significant among mothers with high ethnic-group attachment, $B=-.58, \beta=-.30, t(82)=2.92, p=.088$, trending in the opposite direction (see Figure 1). This pattern suggests that, among newer immigrants, ethnic attachment may buffer the harmful effects of ethnic discrimination on child's health. By comparison, among established immigrant mothers, no significant effects emerged for perceived ethnic discrimination, ethnic-group attachment, or their interaction (see Figure 1).

Table 2

Correlations Between Variables

\begin{tabular}{|c|c|c|c|c|c|c|c|c|c|c|c|}
\hline Variable & 1 & 2 & 3 & 4 & 5 & 6 & 7 & 8 & 9 & 10 & 11 \\
\hline 1. Sick-child visits & - & & & & & & & & & & \\
\hline 2. Ethnic discrimination & .06 & - & & & & & & & & & \\
\hline 3. Language-based discrimination & $.20^{*}$ & $.71^{* * * *}$ & - & & & & & & & & \\
\hline 4. Ethnic-group attachment & $-.21^{*}$ & -.12 & -.14 & - & & & & & & & \\
\hline 5. Years in U.S. & -.05 & -.10 & $-.21^{*}$ & -.13 & - & & & & & & \\
\hline 6. Well-child visits & $-.22^{*}$ & .16 & $.22^{*}$ & .02 & -.13 & - & & & & & \\
\hline 7. Income & -.06 & .09 & -.07 & -.00 & .19 & .12 & - & & & & \\
\hline 8. H.S. education only & -.01 & -.01 & .12 & .10 & $-.26^{*}$ & .01 & -.07 & - & & & \\
\hline 9. College education & -.11 & -.13 & -.14 & .05 & .17 & .18 & .19 & $-.39^{* * * *}$ & - & & \\
\hline 10. Married & -.07 & -.09 & -.04 & -.08 & .11 & .05 & .16 & .00 & -.03 & - & \\
\hline 11. Child age & .07 & .09 & .09 & .06 & -.05 & .09 & $.22^{*}$ & .01 & -.04 & -.15 & - \\
\hline
\end{tabular}

Note. Education variables were categorical variables with mothers who did not complete a high school education coded as 0s. Marital status was a categorical variable as well, with mothers not married to the child's father coded as 0s and mothers married to the child's father coded as 1s.

${ }^{*} p<.05$. *** $p<.001$. 
Table 3

Generalized Estimating Equation Analysis Results Predicting Number of Doctor Visits for Child's Sickness

\begin{tabular}{|c|c|c|c|c|c|c|c|c|}
\hline Variable & $\beta$ & $B$ & $S E$ & $x^{2}$ & $\beta$ & $B$ & $S E$ & $x^{2}$ \\
\hline & \multicolumn{4}{|c|}{ Ethnic Discrimination } & \multicolumn{4}{|c|}{ Language-Based Discrimination } \\
\hline $\begin{array}{l}\text { Predictor variables } \\
\text { (Intercept) }\end{array}$ & & 1068 & 0120 & & & 1057 & 0148 & \\
\hline Discrimination & 0.109 & 0.211 & 0.170 & 1.54 & 0.170 & 0.287 & 0.128 & $5.05^{*}$ \\
\hline Ethnic-group attachment & -0.147 & -0.251 & 0.123 & $4.15^{*}$ & -0.078 & -0.133 & 0.134 & 0.99 \\
\hline Years in U.S. & -0.028 & -0.005 & 0.012 & 0.19 & -0.020 & -0.003 & 0.012 & 0.08 \\
\hline Discrimination $\times$ Ethnic-group attachment & -0.123 & -0.406 & 0.217 & 3.51 & -0.170 & -0.493 & 0.206 & $5.72^{*}$ \\
\hline Discrimination $\times$ Years in U.S. & 0.101 & 0.034 & 0.025 & 1.93 & 0.022 & 0.007 & 0.020 & 0.12 \\
\hline Ethnic-group attachment $\times$ Years in U.S. & 0.108 & 0.032 & 0.021 & 2.44 & 0.098 & 0.029 & 0.023 & 1.57 \\
\hline Discrimination $\times$ Ethnic-group attachment $\times$ Years in U.S. & 0.187 & 0.108 & 0.032 & $11.39^{\text {**** }}$ & 0.183 & 0.093 & 0.029 & $10.46^{* * * * *}$ \\
\hline \multicolumn{9}{|l|}{ Adjustment variables } \\
\hline Income & -0.037 & 0.000 & 0.000 & 0.31 & -0.009 & 0.000 & 0.000 & 0.01 \\
\hline HS education & 0.114 & -0.093 & 0.166 & 0.31 & 0.114 & -0.138 & 0.161 & 0.73 \\
\hline Some college education & 0.306 & -0.222 & 0.211 & 1.10 & 0.306 & -0.267 & 0.225 & 1.42 \\
\hline Married & -0.142 & -0.073 & 0.152 & 0.23 & -0.142 & -0.110 & 0.142 & 0.59 \\
\hline Child doctor visits for wellness & -0.201 & -0.091 & 0.034 & $7.04^{* *}$ & -0.240 & -0.109 & 0.038 & $8.09^{* *}$ \\
\hline Child age & 0.031 & 0.001 & 0.002 & 0.22 & 0.021 & 0.001 & 0.002 & 0.07 \\
\hline Ethnicity & 0.186 & 0.357 & 0.166 & $4.63^{*}$ & 0.186 & 0.390 & 0.177 & $4.85^{*}$ \\
\hline
\end{tabular}

Note. Coefficients are based on two separate equations, one with ethnic discrimination as a predictor, the other with language-based discrimination as a predictor. For both equations all other variables were the same. Mexican mothers who did not complete high school and were not married to the child's father were the reference group. All variables except for the specified child variables refer to mothers. Significant effects are in boldface.

${ }^{*} p<0.5 . \quad{ }^{* * *} p<.01 .{ }^{* * * *} p<.001$

\section{Mothers' Perceived Language-Based Discrimination and Children's Health}

To test the effects of mothers' language-based discrimination on sick-child visits, the model described above was tested again, but with language-based discrimination replacing ethnic discrimination as the main predictor of interest. The GEE analysis revealed a significant main effect for language-based discrimination, indicating that greater perceived discrimination was associated with more frequent sickchild visits. A significant effect also emerged for the discrimination by ethnic-group attachment interaction, but these effects were qualified by the predicted significant three-way interaction (see Table 3 ).

To interpret the observed three-way interaction, the simple interactive effects of perceived language-based discrimination and ethnic-group attachment were again examined separately for recent and established immigrants (i.e., at $+/-1 S D$ relative to the mean values). Among recent immigrants, the expected two-way interaction was significant, $B=-1.02, \beta=-.34, t(80)=8.76$, $p<.01$. That is, greater perceived language-based discrimination predicted more frequent sick-child visits to the doctor among mothers with low ethnic-group attachment, $B=.85, \beta=.49$, $t(80)=10.55, p=.001$, but not for those with high ethnic-group attachment, $B=-.35, \beta=-.19, t(80)=1.60, p=.21$.

Among more established immigrants, only the main effect of language-based discrimination was significant, indicating that more perceived language-based discrimination was associated with an increased number of sick-child visits to the doctor, adjusting for the number of well-child visits, $B=.32, \beta=.20, t(80)=$ 4.05, $p<.05$. See Figure 2 .

\section{Supplementary Analyses}

A set of supplementary analyses were conducted to address potential limitations. First, the extent to which the two types of
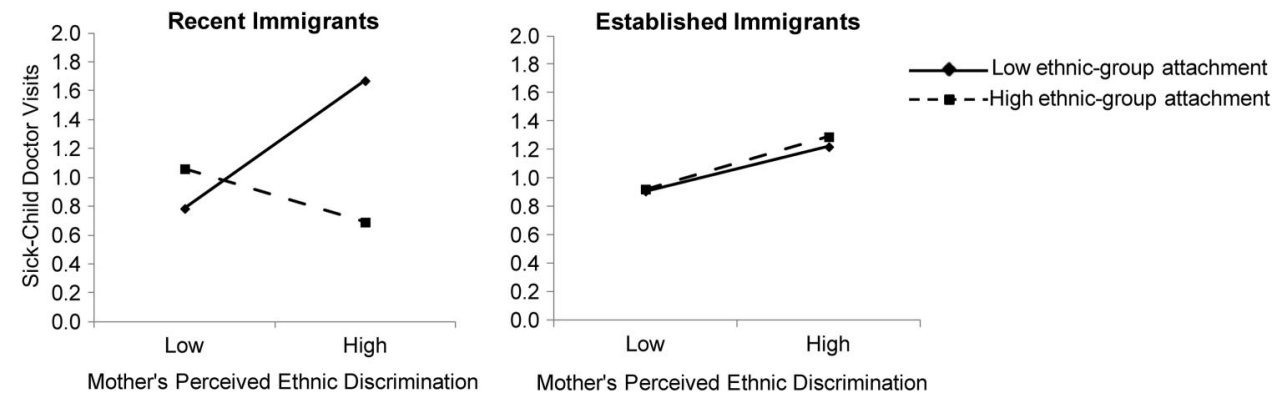

Figure 1. Predicted number of sick-child doctor visits as a function of mother's perceived ethnic discrimination, length of U.S. residency, and ethnic-group attachment. Recent and established immigrants are $1 S D$ above and below the mean of length of U.S. residency. Perceived ethnic discrimination and ethnic-group attachment are plotted at $1 S D$ above and below their respective means. 

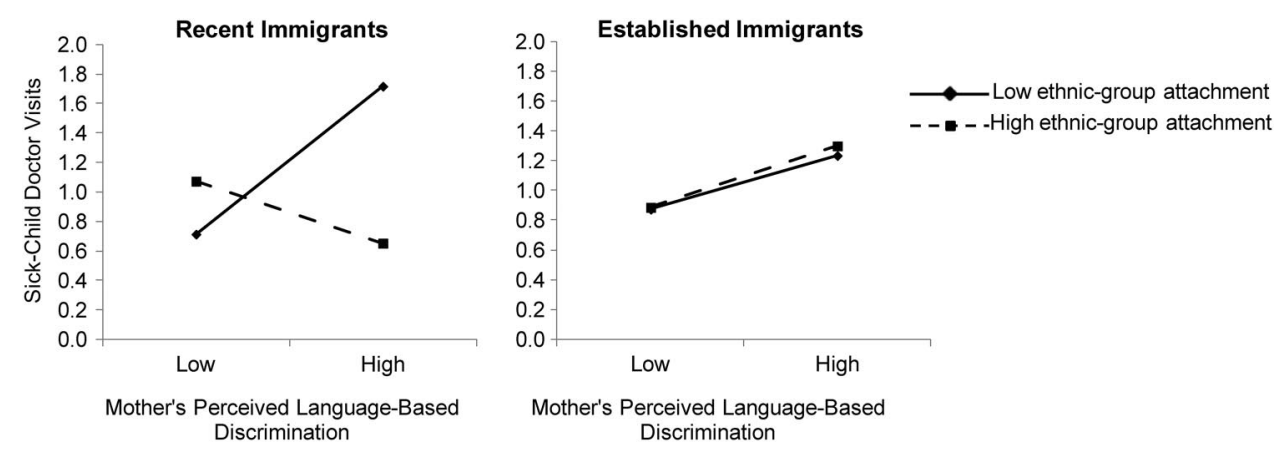

Figure 2. Predicted number of sick-child doctor visits as a function of mother's perceived language-based discrimination, length of U.S. residency, and ethnic-group attachment. Recent and established immigrants are 1 $S D$ above and below the mean of length of U.S. residency. Perceived language-based discrimination and ethnic-group attachment are plotted at $1 S D$ above and below their respective means.

discrimination independently predicted sick-child doctor visits was examined. To this end, the main regression analyses described above were repeated for each form of perceived discrimination while adjusting for the complementary form of perceived discrimination. When language-based discrimination was covaried, the three-way interaction among ethnic discrimination, ethnic-group attachment, and years in the United States predicting sick-child doctor visits remained significant, $B=.102, t(82)=9.63, p=$ .002. Similarly, when ethnic-based discrimination was covaried, the three-way interaction among language-based discrimination, ethnic-group attachment, and years in the United States predicting sick-child doctor visits remained significant, $B=.102, t(82)=$ $10.01, p=.002$.

Analyses were also conducted to demonstrate that the effects of language-based discrimination were not due simply to the participants' actual degree of English language proficiency. To address this issue, the main regression analyses were repeated while adjusting for language use preference (sum of 3 items: "In what language do you sing songs with/read books to/tell stories to your child?"; 0, Spanish only; 1, English and Spanish or English only). Although participants' language proficiency was not assessed directly, this measure provided a proxy. When language use preference was included as a covariate, the three-way interaction among language-based discrimination, ethnic-group attachment, and length of U.S. residency remained a significant predictor of sickchild visits, $B=.091, t(80)=10.52, p=.001$. Thus, the results did not appear to be driven by differences in English language proficiency.

Third, analyses were conducted to further validate that the primary outcome variable - number of sick-child doctor visitscould be interpreted as an indicator of the child's health. To this end, it was important to show that this variable did not simply reflect the mother's utilization of health care services. This issue was addressed in the main analyses by including the number of child's well visits as an adjustment variable. Furthermore, a higher frequency of routine well-child visits, which, in the first year of life, involve receiving vaccines and immunizations, as well as monitoring by a pediatrician, was associated with fewer sick-child visits (see Table 3). It is also notable that greater perceived discrimination has been shown to predict less utilization of health services (Casagrande, Gary, LaVeist, Gaskin, \& Cooper, 2007), but results showed that greater perceived discrimination predicted more sick visits to the doctor. As a complementary approach, mothers' reports of how they typically respond when their child is ill were examined. When asked to consider the child's most recent incident of illness, $18 \%$ of mothers reported that they stayed at home and/or gave home remedies, whereas $82 \%$ reported that they brought their child to see a doctor. Importantly, the tendency to use health care services was not associated with the predictor variables of interest when logistic regressions were conducted with the type of response to illness as the outcome. These results suggest that the outcome variable did indeed provide a valid indicator of children's health and not the mothers' utilization of health care services.

\section{Discussion}

A growing body of research shows that social discrimination has a negative impact on the health of its perceiver (Pascoe \& Richman, 2009). The present findings show that it can also affect on the health of the perceiver's 14-month-old child. Perceptions of discrimination on the basis of language was found to be particularly impactful among the Mexican and Dominican immigrant mothers included in this research, in addition to the perception of ethnicitybased discrimination. Furthermore, the effects of both forms of discrimination on children's health were strongest among more recent immigrants who reported low attachment to their ethnic group. Hence, the deleterious effects of perceived discrimination appear to be mitigated by one's engagement and sense of belonging with other members of one's ethnic group, particularly for newer immigrants. These results provide the first evidence that perceptions of discrimination by a mother could have a negative effect on the health of her child within the first 14 months of her child's life. These findings highlight the impact of social factors on public health while also providing suggestions for how their effects may be ameliorated.

The effects of perceived discrimination on a child's health varied as a function of the mother's degree of ethnic-group attachment and the length of time since her immigration. Ethnic-group attachment was most relevant to recent immigrants, as it strongly moderated the effect of discrimination on children's health among this group. Although the present research did not probe the specific mechanisms underlying this pattern, it is possible that recent 
immigrants are especially dependent on the support of other members of their ethnic group, including family members and neighbors, to cope with discrimination and improve parenting practices and childcare. Indeed, research has found that among Latino women, stronger ethnic identification was associated with stronger familial relations (Deaux \& Martin, 2003).

Among more established immigrants, the impact of perceived discrimination on children's health was weaker, when based on language, or nonsignificant, when based on ethnicity, and the mothers' degree of ethnic attachment did not affect either relationship. The more established immigrants may have had time to build a support network beyond their ethnic group, making this variable less critical. However, the diminished effect of discrimination on children's health among established immigrant mothers is interesting given research showing that, for other health-related variables in other immigrant groups, longer lengths of time in the United States can be a risk factor. For example, in one study, the association between ethnic discrimination and obesity strengthened with increasing years in the United States for Asian immigrants (Gee, Ro, Gavin, \& Takeuchi, 2008). Other research observed a similar pattern for African and Latino immigrants on a range of health outcomes (Gee et al., 2006). In these cases, health risk appeared to increase with their enculturation as minority group members in American society.

A potentially intriguing finding was observed for recent immigrant mothers who reported stronger ethnic-group attachment. Among these mothers, greater perceived discrimination was marginally associated with fewer sick-child doctor visits. A possible explanation for this trend is that for these individuals, experiences of perceived discrimination were met with an outpouring of social support from ingroup members that promoted their child's health. Because this trend was not significant, it should be interpreted with caution. However, it is conceptually consistent with research showing a negative association between perceived ethnic discrimination and alcohol disorders when levels of ethnic identification were high (Chae et al., 2008). Further research is needed to replicate these trends and investigate these explanations.

Although previous work has connected African American mothers' perceived ethnic discrimination with risk for a premature or very low birth weight infant (Giscombe \& Lobel, 2005), the present work suggests that additional research is needed to understand how social factors affect the health of Latino Americans. Latinos represent the fastest-growing segment of ethnic and racial minorities in America (U.S. Census Bureau, 2011), and a recent study found that $30-35 \%$ report experiences with discrimination (Perez, Fortuna, \& Alegria, 2008). The emerging literature linking discrimination with health suggests that a better understanding of discrimination effects among Latino Americans will be critical to addressing public health among a major segment of society.

In addition, this research highlights the importance of languagebased discrimination and suggests it to be a phenomenon distinct from the more frequently studied ethnic discrimination. Languagebased discrimination is especially relevant to the experience of minority-group immigrants who do not speak the host country's dominant language. This study is the first to link greater languagebased discrimination perceived by mothers to poorer health in their children and to show that the significant effects of language-based and ethnicity-based forms of discrimination are distinct. Thus, language-based and ethnic-based forms of discrimination appear to reflect two related yet unique constructs, at least for Latina U.S immigrants. It is notable that although the current sample was primarily Spanish-speaking, some mothers were proficient speakers of English (albeit with an accent). Yet, despite their proficiency, these mothers also reported language-based discrimination on average. Although actual discrimination could not be assessed in this study, this pattern suggests that even immigrants who speak the host language may be affected by language-based discrimination.

The present findings are relevant to policymakers and health care providers, as they suggest that system-wide discrimination, and the consequent perceptions of discrimination, put children's health at risk, but that ethnic-group attachment may buffer this effect. Thus, interventions should focus on two broad issues: reducing system-wide discrimination (e.g., by increasing the diversity of the health care workforce and promoting cultural competence training) and promoting the building of strong community ties among recent immigrants.

The finding that the effects of discrimination can be passed on from mother to child raises questions about the psychological and biological mechanisms through which this occurs. One possibility is that the mother's stress response to perceived discrimination affected the biological development of the child in a way that weakened the child's immune response. Although the present effects on children's health were observed at 14 months, this process could have begun during pregnancy or around the time of birth. A heightened chronic stress response may have influenced children's health prenatally, whether through the levels of stressrelated hormones surrounding the fetus (Rich-Edwards \& Grizzard, 2005) or through teratogens caused by risky health behaviors during pregnancy (Bennett et al., 2010). For example, low birth weight, an outcome of these teratogens, has been associated with increased health risks and developmental problems (McCormick et al., 1992). It follows, then, that children who are less healthy as newborns may continue to be less healthy at 14-months of age and beyond - a hypothesis requiring a longitudinal investigation.

It is also possible that increased stress caused by discrimination could interfere with good parenting practices. Perceived discrimination has been associated with increased negative mood in parents and negative parent-child interactions characterized by less parental warmth, communication, monitoring, and use of reasoning with the child (Broudy et al., 2007; Murry et al., 2001). Concerns about discrimination could distract mothers from caregiving activities that promote good health in the child or make them less likely to seek health care for themselves, which could, in turn, influence the child's health. A different possibility is that perceived discrimination may increase the mother's sensitivity to signs of illness in her child. Although the current study cannot determine the specific underlying mechanism (or mechanisms), it sets the stage for future research on these potential explanations.

A potential limitation in this study was the reliance on mothers' retrospective reports of sick-child visits to indicate their child's health. Such reports could be subject to memory errors. Although there were several factors supporting the validity of this index, a direct objective measure of health, such as data obtained from medical records, would have been more precise. Moreover, the measures used in this study did not assess more nuanced variables, such as the threshold at which a mother decided her child's physical symptoms indicated illness or warranted a doctor's visit. 
It is also possible that the distinction between sick- and well-child visits may have been somewhat unclear to mothers and/or health care providers, although there was no evidence for such confusion in the sample. Nevertheless, to the extent that the reported index of child-sick visits was imprecise, it would have obscured the relation between perceived discrimination and child's health, reducing the strength of the present results. Despite these potential limitations, the current research revealed a novel and meaningful relationship between a mother's perception of discrimination and the health of her offspring.

Another potential limitation was that information on mothers' immigrant documentation status and possession of health care insurance was unavailable. ${ }^{2}$ These factors could affect child doctor visits for illness through their associations with the variables of interest. For example, undocumented immigrants may feel ethnic and language-based discrimination more keenly if they have fewer resources to cope with the challenges of immigration, relative to documented immigrants. However, documentation would likely have affected the frequency of healthy checkups in addition to doctor visits for illness. Given that the results remained significant after adjusting for the frequency of well-child visits, it is unlikely that these findings are attributable to participants' documentation or health care status.

As immigrant families continue to arrive in the United States and elsewhere worldwide, it is increasingly important to study the links between discrimination and health. The findings of the present study suggest that when immigrant women face discrimination based on their ethnicity or the way they speak, their children's health can be at risk. However, feeling a close attachment to an ethnic community may be particularly important for newly arrived immigrant women in buffering the health risks associated with discrimination. The current findings are relevant to the health of Latino immigrant families, an important and urgent topic in the health field (Flores et al., 2002). More broadly, these findings demonstrate the strong link between social factors and health.

\footnotetext{
${ }^{2}$ Documentation status was assessed indirectly, as opposed to directly, (in compliance with IRB protocol) by asking whether anyone in participants' households had possessed or could obtain a savings account, checking account, credit card, and drivers' license (Yoshikawa \& Kholoptseva, 2012). About $24 \%$ of the sample were from households that did not and could not possess any of the 4 items, which can be inferred to represent undocumented immigrant status; another $10 \%$ only possessed 1 item; $10 \%$ possessed 2 items, 20\% possessed 3 items. About 38\% possessed all 4 items, which can be inferred to represent documented immigrant status. Mexican families $(M=1.70, S D=1.71)$ lacked more resources requiring identification than did Dominican families $(M=3.21, S D=1.05), t(93)=$ $5.05, p<.001$. Also, qualitative interviews with a few families in the current study suggest that most of the Mexican mothers were undocumented and most of the Dominican mothers were documented (see Yoshikawa \& Kholoptseva, 2012).
}

\section{References}

Bennett, I. A., Culhane, J. F., Webb, D. A., Coyne, J. C., Hogan, V., Mathew, L., \& Elo, I. T. (2010). Perceived discrimination and depressive symptoms, smoking, and recent alcohol use in pregnancy. Birth, 37, 90-97. doi:10.1111/j.1523-536X.2010.00388.x

Broudy, R., Brondolo, E., Coakley, V., Brady, N., Cassells, A., Tobin,
J. N., \& Sweeney, M. (2007). Perceived ethnic discrimination in relation to daily moods and negative social interactions. Journal of Behavioral Medicine, 30, 31-43. doi:10.1007/s10865-006-9081-4

Campos, B., Schetter, C. D., Abdou, C. M., Hobel, C. J., Glynn, L. M., \& Sandman, C. A. (2008). Familism, social support, and stress: Positive implications for pregnant Latinas. Cultural Diversity and Ethnic Minority Psychology, 14, 155-162. doi:10.1037/1099-9809.14.2.155

Casagrande, S. S., Gary, T. L., LaVeist, T. A., Gaskin, D. J., \& Cooper, L. A. (2007). Perceived discrimination and adherence to medical care in a racially integrated community. Journal of General Internal Medicine, 22, 389-395. doi:10.1007/s11606-006-0057-4

Chae, D. H., Takeuchi, D. T., Barbeau, E. M., Bennett, G. G., Lindsey, J. C., \& Stoddard, A. M. (2008). Alcohol disorders among Asian Americans: Associations with unfair treatment, racial/ethnic discrimination, and ethnic identification (the national Latino and Asian American study, 2002-2003). Journal of Epidemiology and Community Health, 62, 973-979. doi:10.1136/jech.2007.066811

Deaux, K., \& Martin, D. (2003). Interpersonal networks and social categories: Specifying levels of context in identity processes. Social Psychology Quarterly, 66, 101-117. doi:10.2307/1519842

Deaux, K. (2006). To be an immigrant. New York, NY: Russell Sage Foundation.

de Snyder, V. N. S. (1987). Factors associated with acculturative stress and depressive symptomatology among married Mexican immigrant women. Psychology of Women Quarterly, 11, 475-488. doi:10.1111/j.14716402.1987.tb00919.x

Dominguez, T. P., Dunkel-Schetter, C., Glynn, L. M., Hobel, C. J., \& Sandman, C. A. (2008). Racial differences in birth outcomes: The role of general, pregnancy, and racism stress. Health Psychology, 27, 194-203. doi:10.1037/0278-6133.27.2.194

Finch, B. K., Hummer, R. A., Kolody, B., \& Vega, W. A. (2001). The role of discrimination and acculturative stress in the physical health of Mexican-origin adults. Hispanic Journal of Behavioral Sciences, 23, 399-429. doi:10.1177/0739986301234004

Finch, B. K., \& Vega, W. A. (2003). Acculturation stress, social support, and self-rated health among Latinos in California. Journal of Immigrant Health, 5, 109-117.

Flores, G., Fuentes-Afflick, E., Barbot, O., Carter-Pokras, O., Claudio, L., Lara, M., ... Weitzman., M (2002). The hea.lth of Latino children: Urgent priorities, unanswered questions, and a research agenda Journal. of the American Medical Association, 288, 82-90. doi:10.1001/ jama.288.1.82

Gee, G. C., Ro, A., Gavin, A., \& Takeuchi, D. T. (2008). Disentangling the effects of racial and weight discrimination on BMI and obesity. American Journal of Public Health, 98, 493-500. doi:10.2105/ AJPH.2007.114025

Gee, G. C., Ryan, A., Laflamme, D. J., \& Holt, J. (2006). The relationship between perceived discrimination and mental health among African descendents, Mexican Americans and other Latinos in the New Hampshire REACH study: The added dimension of immigration status. American Journal of Public Health, 96, 1821-1828. doi:10.2105/ AJPH.2005.080085

Giscombe, C. L., \& Lobel, M. (2005). Explaining disproportionately high rates of adverse birth outcomes among African Americans: The impact of stress, racism, and related factors in pregnancy. Psychological Bulletin, 5, 662-683. doi:10.1037/0033-2909.131.5.662

Hayes-Bautista, D. E. (2002). The Latino health research agenda for the twenty-first century. In M. M. Suarez-Orozco \& M. Paez (Eds.), Latinos remaking America (pp. 215-235). Berkeley, CA: University of California Press.

Jasinskaja-Lahti, Inga, Liebkind, K., Jaakkola, M., \& Reuter, A. (2006). Perceived discrimination, social support networks, and psychological well-being among three immigrant groups. Journal of Cross-Cultural Psychology, 37, 293-311. doi:10.1177/0022022106286925 
Kim, O. (1999). Mediation effect of social support between ethnic attachment and loneliness in older Korean immigrants. Research in Nursing and Health, 22, 169-175. doi:10.1002/(SICI)1098-240X(199904)22: $2<169:: A I D-N U R 8>3.0 . C O ; 2-F$

McCormick, M. C., Brooks-Gunn, J., Workman-Daniels, K., Turner, J., \& Peckham, G. J. (1992). The health and developmental status of very lowbirth-weight children at school age. The Journal of the American Medical Association, 267, 2204-2208. doi:10.1001/jama.1992.03480160062035

Moradi, B., \& Risco, C. (2006). Perceived discrimination experiences and mental health of Latina/o American persons. Journal of Counseling Psychology, 53, 411-421. doi:10.1037/0022-0167.53.4.411

Murry, V. M., Brown, P. A., Brody, G. H., Cutrona, C. E., \& Simons, R. L. (2001). Racial discrimination as a moderator of the links among stress, maternal psychological functioning, and family relationships. Journal of Marriage and Family, 63, 915-926. doi:10.1111/j.1741-3737.2001.00915.x

Pascoe, E. A., \& Richman, L. S. (2009). Perceived discrimination and health: A meta-analytic review. Psychological Bulletin, 125, 531-534. doi:10.1037/a0016059

Perez, D. J., Fortuna, L., \& Alegria, M. (2008). Prevalence and correlates of everyday discrimination among U.S. Latinos. Journal of Community Psychology, 36, 421-433. doi:10.1002/jcop.20221

Phillips-Howard, P. A., Nahlen, B. L., Wannemuehler, K. A., Kolczak, M. S., Ter Kuile, F. O., Gimnig, J. E., \& Hawley, W. A. (2003). Impact of permethrin-treated bed nets on the incidence of sick child visits to peripheral health facilities. American Journal of Tropical Medicine and Hygiene, 68, 38-43.

Phinney, J. S. (1992). The multigroup ethnic identity measure: A new scale for use with diverse groups. Journal of Adolescent Research, 7, 156176. doi: $10.1177 / 074355489272003$

Rich-Edwards, J. W., \& Grizzard, T. A. (2005). Psychosocial stress and neuroendocrine mechanisms in preterm delivery. American Journal of $\mathrm{Ob}$ stetrics and Gynecology, 192, S30-S35. doi:10.1016/j.ajog.2005.01.072

Spencer, M. S., \& Chen, J. (2004). Effect of discrimination on mental health service utilization of among Chinese Americans. American Journal of Public Health, 94, 809-814. doi:10.2105/AJPH.94.5.809

Stuber, J., Galea, S., Ahern, J., Blaney, S., \& Fuller, C. (2003). The association between multiple domains of discrimination and selfassessed health: A multilevel analysis of Latinos and Blacks in four low-income New York City neighborhoods. Health Services Research, 38, 1735-1760. doi:10.1111/j.1475-6773.2003.00200.x

Timmins, C. L. (2002). The impact of language barriers on the health care of Latinos in the United States: A review of the literature and guidelines for practice. Journal of Midwifery and Women's Health, 47, 80-96. doi:10.1016/S1526-9523(02)00218-0

U.S. Census Bureau. (2011). Statistical abstract of the United States, Population. Retrieved April 13, 2011, from http://www.census.gov/ compendia/statab/2011/tables/11s0005.pdf

Williams, D. R., Yu, Y., Jackson, J. S., \& Anderson, N. B. (1997). Racial differences in physical and mental health: Socioeconomic status, stress and discrimination. Journal of Health Psychology, 2, 335-351. doi: $10.1177 / 135910539700200305$

Wright, P. F., Thompson, J., Mckee, K. T., Vaughn, W. K., Sell, S. H. W. \& Karzon, D. T. (1981). Patterns of illness in the highly febrile young child: Epidemiological, clinical, and laboratory correlates. Pediatrics, 67, 694-700.

Wright, R. J. (2011). Epidemiology of stress and asthma: From constricting communities and fragile families to epigenetics. Immunology and Allergy Clinics of North America, 31, 19-39. doi:10.1016/j.iac.2010.09.011

Yip, T., Gee, G. C., \& Takeuchi, D. T. (2008). Racial discrimination and psychological distress: The impact of ethnic identity and age among immigrant and United-States born Asian adults. Developmental Psychology, 44, 787-800. doi:10.1037/0012-1649.44.3.787

Yoo, H. C., Gee, G. C., \& Takeuchi, D. (2009). Discrimination and health among Asian American immigrants: Disentangling racial from language discrimination. Social Science \& Medicine, 68, 726-732. doi:10.1016/ j.socscimed.2008.11.013

Yoshikawa, H., \& Kholoptseva, J. (2012). Everyday experiences of being an undocumented immigrant parent: Associations with early child learning. Manuscript under review.

Yoshikawa, H., Wilson, P. A., Chae, D. H., \& Cheng. J. (2004). Do family and friendship networks protect against the influence of discrimination on mental health and HIV risk among Asian and Pacific Islander gay men? AIDS Education and Prevention, 16, 84-100. doi:10.1521/ aeap.16.1.84.27719

Zeger, S. L., Liang, K., \& Albert, P. S. (1988). Models for longitudinal data: A generalized estimating equation approach. Biometrics, 44, 1049-1060. doi:10.2307/2531734

Received April 27, 2011

Revision received December 30, 2011

Accepted December 30, 2011 